\title{
TRACES OF HADAMARD AND KRONECKER PRODUCTS OF MATRICES
}

\author{
PANKAJ KUMAR DAS AND LALIT K. VASHISHT
}

\begin{abstract}
We present some inequality/equality for traces of Hadamard product and Kronecker product of matrices. Some numerical examples are given to support the results.
\end{abstract}

\section{INTRODUCTION}

The Hadamard (or Schur) and Kronecker products are widely studied and applied in matrix theory, statistics, system theory and other areas [2]. It was Schur who initially studied algebraic and analytic properties of Hadamard product. In 1990, Horn [1] presents a widespread information focusing on the Hadamard product. Magnus and Neudecker [4] give some basic results and statistical applications involving Hadamard or Kronecker products. For basics on these two matrix products, one may refer to [3,5-7]. In [6], the authors investigated traces of Hadamard and Kronecker products of matrices and obtained some inequalities for traces of products of matrices. In this note, we present some inequality for traces of Hadamard product, Kronecker product and mixed type product of matrices.

We recall the basic definitions and notations to make our presentation selfcontained. The set of all positive real numbers is denoted by $\mathbb{R}^{+}$. By $M_{n}$ we denote the family of $n$-by- $n$ matrices over the real field $\mathbb{R}$. For $A=\left[a_{i j}\right] \in M_{n}$, the scalar $\sum_{i=1}^{n} a_{i i}$ is called the trace of $A$ and is usually denoted by $\operatorname{tr}(A)$ or $\operatorname{trace} A$. The Hadamard product of two matrices $A=\left[a_{i j}\right]$ and $B=\left[b_{i j}\right]$ of identical size is just their element-wise product which is given by

$$
A \circ B=\left[a_{i j} b_{i j}\right]_{m \times n} .
$$

Let $A=\left[a_{i j}\right]$ be an $m$-by- $n$ matrix and let $B=\left[b_{i j}\right]$ be a $p$-by- $q$ matrix. The Kronecker product of $A$ and $B$ is defined as

$$
A \otimes B=\left[\begin{array}{cccc}
a_{11} B & a_{12} B & \ldots & a_{1 n} B \\
a_{21} B & a_{22} B & \ldots & a_{2 n} B \\
\vdots & \vdots & \ddots & \vdots \\
a_{m 1} B & a_{m 2} B & \ldots & a_{m n} B
\end{array}\right]
$$

$M S C$ (2010): primary 15A45; secondary $15 \mathrm{~A} 24$.

Keywords: trace, Hadamard product, Kronecker product. 
Lemma 1.1. [6] Let $A \in M_{n}$ and $B \in M_{m}$. Then,

$$
\operatorname{tr}(A \otimes B)=\operatorname{tr}(B \otimes A)=\operatorname{tr}(A) \operatorname{tr}(B) .
$$

Theorem 1.2. [6] If $A=\left[a_{i j}\right], B=\left[b_{i j}\right] \in M_{n}$, then

(1) $\operatorname{tr}(A \otimes B)=n \operatorname{tr}(A \circ B)-\sum_{i=1}^{n-1} \sum_{j=i+1}^{n}\left(a_{i i}-a_{j j}\right)\left(b_{i i}-b_{j j}\right)$.

(2) $\operatorname{tr}(A \circ B) \leq \operatorname{tr}\left(\left(\frac{A+B}{2}\right) \circ\left(\frac{A+B}{2}\right)\right)$.

\section{MAin Results}

We start with an inequality involving the trace of $m$ times Hadamard product of a matrix and the trace of the given matrix .

Proposition 2.1. Let $A \in M_{n}$ with positive real diagonal. Then,

$$
\operatorname{tr}(\underbrace{A \circ A \circ A \circ \cdots \circ A}_{m}) \leq(\operatorname{tr}(A))^{m} .
$$

Proof. Let $A=\left[a_{i j}\right] \in M_{n}$ and let $a_{i i} \in \mathbb{R}^{+}$. Then, by the definitions of Hadamard product and trace of matrices, we have

$$
\operatorname{tr}(\underbrace{A \circ A \circ A \circ \cdots \circ A}_{m})=\sum_{i=1}^{n} a_{i i}^{m} \leq\left(\sum_{i=1}^{m} a_{i i}\right)^{m}=(\operatorname{tr}(A))^{m} .
$$

The proposition is proved.

Proposition 2.2. If $A=\left[a_{i j}\right], B=\left[b_{i j}\right] \in M_{n}$, then

$$
\begin{aligned}
n \operatorname{tr}((\underbrace{A \circ A \circ \cdots \circ A}_{n}) \circ(\underbrace{B \circ B \circ \cdots \circ B}_{n})) \\
=\sum_{i=1}^{n} a_{i i}^{n} \sum_{i=1}^{n} b_{i i}^{n}-\sum_{i=1}^{n-1} \sum_{j=i+1}^{n}\left(a_{i i}^{n}-a_{j j}^{n}\right)\left(b_{i i}^{n}-b_{j j}^{n}\right) .
\end{aligned}
$$

Proof. Since

$$
\operatorname{tr}((\underbrace{A \circ A \circ \cdots \circ A}_{n}) \otimes(\underbrace{B \circ B \circ \cdots \circ B}_{n}))=\sum_{i=1}^{n} a_{i i}^{n} \sum_{i=1}^{n} b_{i i}^{n},
$$

applying Theorem 1 in [6], we get

$$
\begin{aligned}
n \operatorname{tr}((\underbrace{A \circ A \circ \cdots \circ A}_{n}) \circ \underbrace{(B \circ B \circ \cdots \circ B}_{n}) & \\
& =\sum_{i=1}^{n} a_{i i}^{n} \sum_{i=1}^{n} b_{i i}^{n}-\sum_{i=1}^{n-1} \sum_{j=i+1}^{n}\left(a_{i i}^{n}-a_{j j}^{n}\right)\left(b_{i i}^{n}-b_{j j}^{n}\right) .
\end{aligned}
$$

The following proposition provides a relation between the trace of a matrix and Kronecker product of the given matrix. 
Proposition 2.3. For any $A \in M_{n}$, we have

$$
\operatorname{tr}(\underbrace{A \otimes A \otimes A \otimes \cdots \otimes A}_{m})=(\operatorname{tr}(A))^{m} .
$$

Proof. We prove the proposition by induction. By Lemma 1.1, the equation is true for $n=2$. Assume that it is true $n=k$, i.e.,

$$
\operatorname{tr}(\underbrace{A \otimes A \otimes A \otimes \cdots \otimes A}_{k})=(\operatorname{tr}(A))^{k} .
$$

Again using Lemma 1.1, we compute

$$
\begin{aligned}
\operatorname{tr}(\underbrace{A \otimes A \otimes A \otimes \cdots \otimes A}_{k+1}) & =\operatorname{tr}(\underbrace{A \otimes A \otimes A \otimes \cdots \otimes A}_{k}) \operatorname{tr}(A) \\
& =(\operatorname{tr}(A))^{k} \operatorname{tr}(A) \quad(\text { by }(2.1)) \\
& =(\operatorname{tr}(A))^{k+1} .
\end{aligned}
$$

The proposition is proved.

Example 2.4. Let $A=\left[\begin{array}{ll}3 & 1 \\ 4 & 1\end{array}\right]$. Then,

$$
A \circ A=\left[\begin{array}{cc}
9 & 1 \\
16 & 1
\end{array}\right], \quad A \otimes A \otimes A=\left[\begin{array}{cccccccc}
27 & 9 & 9 & 3 & 9 & 3 & 3 & 1 \\
36 & 9 & 12 & 3 & 12 & 3 & 4 & 1 \\
36 & 12 & 9 & 3 & 12 & 4 & 3 & 1 \\
48 & 12 & 12 & 3 & 16 & 4 & 4 & 1 \\
36 & 12 & 12 & 4 & 9 & 3 & 3 & 1 \\
48 & 12 & 16 & 4 & 12 & 3 & 4 & 1 \\
48 & 16 & 12 & 4 & 12 & 4 & 3 & 1 \\
64 & 16 & 16 & 4 & 16 & 4 & 4 & 1
\end{array}\right]
$$

and $\operatorname{tr}(A)=4$. Now we have $\operatorname{tr}(A \circ A)=10 \leq 4^{2}=(\operatorname{tr}(A))^{3}$. So, Proposition 2.1 is true. Also, $\operatorname{tr}(A \otimes A \otimes A)=64=(\operatorname{tr}(A))^{3}$. This verifies Proposition 2.2.

Proposition 2.5. For any $A \in M_{n}$, we have

$$
\operatorname{tr}\left(\left(A+A^{t}\right) \circ\left(A+A^{t}\right)\right)=4 \operatorname{tr}(A \circ A) .
$$

Proof. Let $A=\left[a_{i j}\right] \in M_{n}$. Then

$\operatorname{tr}\left(\left(A+A^{t}\right) \circ\left(A+A^{t}\right)\right)=\sum_{i=1}^{n}\left(a_{i i}+a_{i i}\right)\left(a_{i i}+a_{i i}\right)=4 \sum_{i=1}^{n} a_{i i}^{2}=4 \operatorname{tr}(A \circ A)$.

The proposition is proved.

Proposition 2.6. For any $A, B \in M_{n}$, we have

$$
\operatorname{tr}((A+B) \circ(A-B))=\operatorname{tr}(A \circ A)-\operatorname{tr}(B \circ B) .
$$


Proof. Let $A=\left[a_{i j}\right], B=\left[b_{i j}\right] \in M_{n}$ be arbitrary.

We compute

$$
\begin{aligned}
\operatorname{tr}((A+B) \circ(A-B)) & =\sum_{i=1}^{n}\left(a_{i i}+b_{i i}\right)\left(a_{i i}-b_{i i}\right) \\
& =\sum_{i=1}^{n}\left(\left(a_{i i}\right)^{2}-\left(b_{i i}\right)^{2}\right) \\
& =\operatorname{tr}(A \circ A)-\operatorname{tr}(B \circ B) .
\end{aligned}
$$

Proposition 2.7. For any $A, B \in M_{n}$, we have

$$
\operatorname{tr}((A+B) \circ(A+B))=\operatorname{tr}(A \circ A)+2 \operatorname{tr}(A \circ B)+\operatorname{tr}(B \circ B) .
$$

Proof. Similar to proof of Proposition 2.6.

The following proposition gives the relationship between trace of a matrix obtained as Kronecker product of a finite sum of matrices and the traces of the matrices. We prove the result for two matrices.

Proposition 2.8. Let $A, B \in M_{n}$. Then

$$
\begin{array}{r}
\operatorname{tr}(\underbrace{(A+B) \otimes(A+B) \otimes(A+B) \otimes \cdots \otimes(A+B)}_{k}) \\
=\sum_{i=1}^{k}\left(\begin{array}{c}
k \\
i
\end{array}\right)(\operatorname{tr}(A))^{k-i}(\operatorname{tr}(B))^{i} .
\end{array}
$$

Proof. Using Proposition 2.3, we compute

$$
\begin{aligned}
& \operatorname{tr}(\underbrace{(A+B) \otimes(A+B) \otimes(A+B) \otimes \cdots \otimes(A+B)}_{k}) \\
= & (\operatorname{tr}(A+B))^{k}=(\operatorname{tr}(A)+\operatorname{tr}(B))^{k} \\
= & \sum_{i=1}^{k}\left(\begin{array}{l}
k \\
i
\end{array}\right)(\operatorname{tr}(A))^{k-i}(\operatorname{tr}(B))^{i} .
\end{aligned}
$$

The result is proved.

Remark 2.9. Using Proposition 2.1, for any $A, B \in M_{n}$ with positive diagonal, we can show that

$$
\begin{aligned}
\operatorname{tr}(\underbrace{(A+B) \circ(A+B) \circ(A+B) \circ \cdots \circ(A+B)}_{n}) \\
\leq \sum_{i=1}^{n}\left(\begin{array}{c}
n \\
i
\end{array}\right)(\operatorname{tr}(A))^{n-i}(\operatorname{tr}(B))^{i} .
\end{aligned}
$$


The next proposition gives a trace inequality for the Hadamard product of matrix sums.

Proposition 2.10. If $A, B \in M_{n}$, then

$$
(\operatorname{tr}(A \circ B))^{2} \leq \frac{1}{16} \operatorname{tr}((A+B) \circ(A+B) \circ(A+B) \circ(A+B)) .
$$

Proof. Using Theorem 1.2 and Proposition 2.3, we compute

$$
\begin{aligned}
(\operatorname{tr}(A \circ B))^{2} & \leq \operatorname{tr}\left(\left(\frac{A+B}{2}\right) \circ\left(\frac{A+B}{2}\right)\right) \operatorname{tr}\left(\left(\frac{A+B}{2}\right) \circ\left(\frac{A+B}{2}\right)\right) \\
& =\operatorname{tr}\left(\left(\frac{A+B}{2}\right) \circ\left(\frac{A+B}{2}\right) \circ\left(\frac{A+B}{2}\right) \circ\left(\frac{A+B}{2}\right)\right) \\
& =\frac{1}{16} \operatorname{tr}((A+B) \circ(A+B) \circ(A+B) \circ(A+B)) .
\end{aligned}
$$

The proposition is proved.

The following theorem provides a relation between the trace of matrices generated by Kronecker and Hadamard product of matrices.

Theorem 2.11. If $A, B, C, D \in M_{n}$, then

(1) $(A \otimes B) \circ(C \otimes D)=(A \circ C) \otimes(B \circ D)$.

(2) $\operatorname{tr}(A \otimes(B \circ C))=n \operatorname{tr}(A \circ B \circ C)-\sum_{i=1}^{n-1} \sum_{j=i+1}^{n}\left(a_{i i}-a_{j j}\right)\left(b_{i i} c_{i i}-b_{j j} c_{j j}\right)$.

Proof. Let us write $A=\left[a_{i j}\right]$ and $C=\left[c_{i j}\right]$. Then, we compute

$$
\begin{aligned}
& (A \otimes B) \circ(C \otimes D) \\
= & {\left[\begin{array}{cccc}
a_{11} B & a_{12} B & \ldots & a_{1 n} B \\
a_{21} B & a_{22} B & \ldots & a_{2 n} B \\
\vdots & \vdots & \ddots & \vdots \\
a_{n 1} B & a_{n 2} B & \ldots & a_{n n} B
\end{array}\right] \circ\left[\begin{array}{cccc}
c_{11} D & c_{12} D & \ldots & c_{1 n} D \\
c_{21} D & c_{22} D & \ldots & c_{2 n} D \\
\vdots & \vdots & \ddots & \vdots \\
c_{n 1} D & c_{n 2} D & \ldots & c_{n n} D
\end{array}\right] } \\
= & {\left[\begin{array}{cccc}
a_{11} c_{11} B \circ D & a_{12} c_{12} B \circ D & \ldots & a_{1 n} c_{1 n} B \circ D \\
a_{21} c_{21} B \circ D & a_{22} c_{22} B \circ D & \ldots & a_{2 n} c_{2 n} B \circ D \\
\vdots & \vdots & \ddots & \vdots \\
a_{n 1} c_{n 1} B \circ D & a_{n 2} c_{n 2} B \circ D & \ldots & a_{n n} c_{n n} B \circ D
\end{array}\right]=(A \circ C) \otimes(B \circ D) . }
\end{aligned}
$$

Hence, (1) is proved.

Let us write $B=\left[b_{i j}\right]$. Then, by Theorem 1.2 , we have

$$
\begin{aligned}
\operatorname{tr}(A \otimes(B \circ C)) & =n \operatorname{tr}(A \circ(B \circ C))-\sum_{i=1}^{n-1} \sum_{j=i+1}^{n}\left(a_{i i}-a_{j j}\right)\left(b_{i i} c_{i i}-b_{j j} c_{j j}\right) \\
& =n \operatorname{tr}(A \circ B \circ C)-\sum_{i=1}^{n-1} \sum_{j=i+1}^{n}\left(a_{i i}-a_{j j}\right)\left(b_{i i} c_{i i}-b_{j j} c_{j j}\right) .
\end{aligned}
$$

Thus, (2) is proved. 
Corollary 2.12. If $A, B, C, D \in M_{n}$, then

$$
\begin{aligned}
\operatorname{tr}((A \otimes B) \circ(C \otimes D))=n \operatorname{tr}(A \circ B \circ C \circ D) & \\
& -\sum_{i=1}^{n-1} \sum_{j=i+1}^{n}\left(a_{i i} c_{i i}-a_{j j} c_{j j}\right)\left(b_{i i} d_{i i}-b_{j j} d_{j j}\right) .
\end{aligned}
$$

Indeed, by Theorem 2.11, we can write

$$
\operatorname{tr}((A \otimes B) \circ(C \otimes D))=\operatorname{tr}((A \circ C) \otimes(B \circ D)) .
$$

Thus, by Theorem 1.2, we have

$$
\begin{aligned}
\operatorname{tr}((A \otimes B) \circ(C \otimes D))=n \operatorname{tr}(A \circ C \circ B \circ D) & \\
& -\sum_{i=1}^{n-1} \sum_{j=i+1}^{n}\left(a_{i i} c_{i i}-a_{j j} c_{j j}\right)\left(b_{i i} d_{i i}-b_{j j} d_{j j}\right) .
\end{aligned}
$$

Remark 2.13. If $A, C \in M_{n}$ and $B, D \in M_{k}(k \neq n)$, then the equations given in Theorem 2.11 hold too.

Example 2.14. Let $A=\left[\begin{array}{cc}3 & 1 \\ 4 & -1\end{array}\right], B=\left[\begin{array}{cc}4 & -1 \\ 3 & 2\end{array}\right], C=\left[\begin{array}{cc}2 & 3 \\ 1 & -1\end{array}\right]$ and $D=\left[\begin{array}{cc}-2 & 1 \\ -1 & -3\end{array}\right]$

Then

$$
\begin{gathered}
A \otimes B=\left(\begin{array}{cccc}
12 & -3 & 4 & -1 \\
9 & 6 & 3 & 2 \\
16 & -4 & -4 & 1 \\
12 & 8 & -3 & -2
\end{array}\right), \quad C \otimes D=\left(\begin{array}{cccc}
-4 & 2 & -6 & 3 \\
-2 & -6 & -3 & -9 \\
-2 & 1 & 2 & -1 \\
-1 & -3 & 1 & 3
\end{array}\right) ; \\
A \circ C=\left[\begin{array}{ll}
6 & 3 \\
4 & 1
\end{array}\right], \quad B \circ D=\left[\begin{array}{cc}
-8 & -1 \\
-3 & -6
\end{array}\right] ;
\end{gathered}
$$

and

$$
A \otimes(B \circ D)=\left(\begin{array}{cccc}
-24 & -3 & -8 & -1 \\
-9 & -18 & -3 & -6 \\
-32 & -4 & 8 & 1 \\
-12 & -24 & 3 & 6
\end{array}\right), \quad A \circ B \circ D=\left[\begin{array}{cc}
-24 & -1 \\
-12 & 6
\end{array}\right] .
$$

It is easy to see that

$$
(A \otimes B) \circ(C \otimes D)=\left(\begin{array}{cccc}
-48 & -6 & -24 & -3 \\
-18 & -36 & -9 & -18 \\
-32 & -4 & -8 & -1 \\
-12 & -24 & -3 & -6
\end{array}\right)=(A \circ C) \otimes(B \circ D) .
$$

We compute

$$
n \operatorname{tr}(A \circ B \circ D)-\sum_{i=1}^{n-1} \sum_{j=i+1}^{n}\left(a_{i i}-a_{j j}\right)\left(b_{i i} d_{i i}-b_{j j} d_{j j}\right)
$$




$$
=2(-18)-(-8)(\text { here } n=2)=-28=\operatorname{tr}(A \otimes(B \circ D)) .
$$

Thus, Theorem 2.11 is verified.

To conclude the paper, we give a result that connects the traces of matrices obtained by Kronecker and Hadamard product of matrices in terms of the trace of Hadamard product of matrices.

Theorem 2.15. If $A_{i}, B_{i} \in M_{n}$ for $1 \leq i \leq n$, then

$$
\operatorname{tr}\left(\left(A_{1} \otimes A_{2} \otimes \cdots \otimes A_{n}\right) \circ\left(B_{1} \otimes B_{2} \otimes \cdots \otimes B_{n}\right)\right)=\prod_{i=1}^{n} \operatorname{tr}\left(A_{i} \circ B_{i}\right) .
$$

Proof. We prove the theorem by induction. Clearly, the equation is true for $n=1$. Assume that it is true for $n=k$, i.e.,

$$
\operatorname{tr}\left(\left(A_{1} \otimes A_{2} \otimes \cdots \otimes A_{k}\right) \circ\left(B_{1} \otimes B_{2} \otimes \cdots \otimes B_{k}\right)\right)=\prod_{i=1}^{k} \operatorname{tr}\left(A_{i} \circ B_{i}\right) .
$$

We compute

$$
\begin{aligned}
& \operatorname{tr}\left(\left(A_{1} \otimes A_{2} \otimes \cdots \otimes A_{k+1}\right) \circ\left(B_{1} \otimes B_{2} \otimes \cdots \otimes B_{k+1}\right)\right) \\
= & \operatorname{tr}\left\{\left(\left(A_{1} \otimes A_{2} \otimes \cdots \otimes A_{k}\right) \circ\left(B_{1} \otimes B_{2} \otimes \cdots \otimes B_{k}\right)\right) \otimes\left(A_{k+1} \circ B_{k+1}\right)\right\}
\end{aligned}
$$

(by Theorem 2.11)

$$
=\operatorname{tr}\left(\left(A_{1} \otimes A_{2} \otimes \cdots \otimes A_{k}\right) \circ\left(B_{1} \otimes B_{2} \otimes \cdots \otimes B_{k}\right)\right) \operatorname{tr}\left(A_{k+1} \circ B_{k+1}\right)
$$

(by Lemma 1.1)

$$
\begin{aligned}
& =\prod_{i=1}^{k} \operatorname{tr}\left(A_{i} \circ B_{i}\right) \operatorname{tr}\left(A_{k+1} \circ B_{k+1}\right) \\
& =\prod_{i=1}^{k+1} \operatorname{tr}\left(A_{i} \circ B_{i}\right) .
\end{aligned}
$$

Thus, the equation is true for $n=k+1$. The theorem is proved.

Remark 2.16. Theorem 2.15 is useful because the computation of $A_{i} \circ B_{i}$ $(1 \leq i \leq n)$ is much easier than $\left(A_{1} \otimes A_{2} \otimes \cdots \otimes A_{n}\right) \circ\left(B_{1} \otimes B_{2} \otimes \cdots \otimes B_{n}\right)$.

\section{REFERENCES}

[1] R. A. Horn, The Hadamard product, Proc. Symp. Appl. Math. 40 (1990), 87-169.

[2] R. A. Horn and C. R. Johnson, Matrix Analysis, 2nd ed., Cambridge University Press, 2010.

[3] S. Liu and G. Trenkler, Hadamard, Khatri-Rao, Kronecker and other matrix products, Int. J. Inf. Syst. Sci. 4 (2008), 160-177.

[4] J. R. Magnus and H. Neudecker, Matrix Differential Calculus with Applications in Statistics and Econometrics, 2nd ed., Wiley, Chichester, UK, 1999.

[5] M. Özel, Trace and determinant inequalities for Hadamard products, International Journal of Physical Sciences 7 (2012), 1-4.

[6] N. Taskara and I. H. Gumus, On the traces of Hadamard and Kronecker products of matrices, Selcuk Journal of Applied Mathematics 14 (2013), 31-36. 
[7] H. Zhang and F. Ding, On the Kronecker products and their applications, J. Appl. Math. 2013 (2013), Article ID 296185, 8 pp.

Pankaj Kumar Das, Department of Mathematical Sciences, Tezpur University, Napaam, Tezpur, Assam-784028, India (On lien from Shivaji College, University of Delhi, Department of Mathematics, Raja Garden, Ring Road, New Delhi-110 027, India)

e-mail: pankaj4thapril@yahoo.co.in, pankaj4@tezu.ernet.in

Lalit K. Vashisht, Department of Mathematics, University of Delhi, Delhi-110007, India e-mail: lalitkvashisht@gmail.com 